\title{
Dynamics of semiconductor lasers with bidirectional optoelectronic coupling: Stability, route to chaos, and entrainment
}

\author{
Raúl Vicente, ${ }^{1,2}$ Shuo Tang, ${ }^{2}$ Josep Mulet, ${ }^{1}$ Claudio R. Mirasso, ${ }^{1,2}$ and Jia-Ming Liu ${ }^{2}$ \\ ${ }^{1}$ Department de Física, Universitat de les Illes Balears, Campus UIB, E-07122 Palma de Mallorca, Spain \\ ${ }^{2}$ Department of Electrical Engineering, University of California, Los Angeles, Los Angeles, California 90095-159410, USA
}

(Received 3 March 2004; revised manuscript received 11 May 2004; published 26 October 2004)

\begin{abstract}
The dynamical behavior of two mutually coupled semiconductor lasers is studied. An optoelectronic coupling including a time delay in the propagation of the signals between the two lasers is considered. Starting from the appropriate rate equations for the photon and carrier densities, we investigate the stability of the fixed points and limit cycles of the system as a function of the coupling strength and the propagation time. From this analysis, a quasiperiodic route to chaos with boundary crisis events is identified as the responsible mechanism leading the system from regular to complex behavior. Several interesting phenomena are predicted for this system. Our analytical and numerical results are supported by experiments which are in good agreement with our predictions.
\end{abstract}

DOI: $10.1103 /$ PhysRevE.70.046216

PACS number(s): 05.45.Xt, 42.55.Px, 42.65.Sf, 05.45.Vx

\section{INTRODUCTION}

Neurons, chemical oscillators, Josephson junctions, or semiconductor lasers are just a few examples of nonlinear systems in which the interaction of many similar parts can lead to very rich and unexpected behaviors [1]. Their importance, in both theoretical and practical applications, has attracted the attention of researchers for a long time. While the dynamics of coupled oscillators has been extensively studied, only recently have the effects of finite connection time, which naturally arise from the finite propagation speed of the signals between the subsystems, been taken into account. In mutually interacting subsystems, the delay time not only introduces an infinite-dimensional phase space [2] but also provides a new source of possible instabilities. For their potential implications in physics, medicine, biology, and chemistry, mutually coupled nonlinear oscillators have received much attention.

Semiconductor lasers are ideal candidates for the study of coupled systems since their dynamics is well understood, both theoretically and experimentally, in different situations. Semiconductor lasers subject to optoelectronic feedback have been extensively studied for many years [3-8]. Optoelectronically linked semiconductor lasers [9-12] have been used in unidirectionally coupled schemes with applications to encoded communications [13-15]. However, mutually interacting semiconductor lasers have been only considered, to the best of our knowledge, assuming a coherent optical coupling [16-21]. In all these studies interesting dynamical regimes, including anticipated and retarded synchronization, leader-laggard dynamics, etc., have been reported.

In this paper, we investigate the dynamical properties of two semiconductor lasers subject to a bidirectional optoelectronic coupling. The lasers exhibit continuous wave (cw) operation when they are decoupled. We focus on the instabilities arising from the delayed interaction and the entrainment properties of these instabilities when the coupling strength is enhanced. Although the study we perform here is under the degenerate condition of identical lasers, we remark that this type of system is relevant because of its role as organizing center of the dynamics. This configuration allows us to study the relative dynamics between the two lasers and its dependence on several easily adjustable parameters, an investigation with no counterpart in the case of a single laser subject to delayed optoelectronic feedback. Moreover, the mutual entrainment or synchronization phenomena between the two semiconductor lasers are topics under current research [17-19,21,22] with significant implications in such subjects as optical cryptography and injection locking between lasers.

The choice of the optoelectronic coupling between the lasers allows for a better understanding of the general phenomena in this kind of delayed coupled systems. Moreover, optoelectronic coupling avoids the complexity introduced by the phase of the electric field that otherwise plays a crucial role when considering the optical coupling [17,20,21]. Another advantage of the study of this system comes from the fact that we do not need to restrict ourselves to investigate weak- or moderate-coupling coefficients in order to avoid multiple delayed terms, as happens in the mutual coherent optical interaction, which allows us to explore both the weak- and strong-coupling regimes with a simple set of rate equations. However, we must point out that at a very large coupling strength additional terms that address the saturation effects in photodetectors and amplifiers have to be included in the model.

Our work is organized as follows. In Sec. II we present the model of the system that is developed at the level of modified rate equations. After some general considerations, we present in Secs. III and IV the analytical and numerical results. These are mainly devoted to stability charts, the full explanation of the route to chaos followed by the system, and the study of the mutual entrainment between the two lasers. In Sec. V experimental results that validate our theoretical and numerical studies are presented. Concluding remarks are given in Sec. VI.

\section{MODEL}

We consider the system depicted in Fig. 1, where the optical output power emerging from each laser is converted 


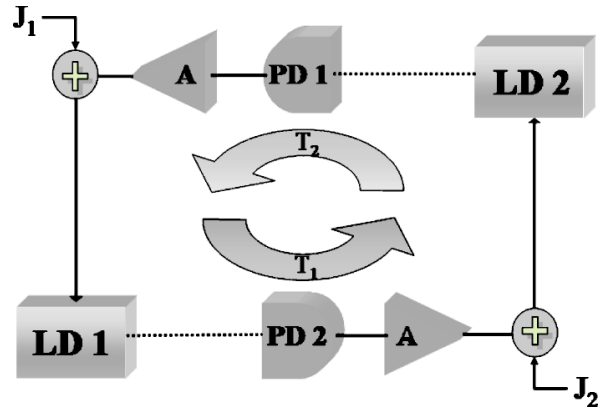

FIG. 1. Scheme of two lasers with optoelectronic bidirectional coupling.

into photocurrent and, after an amplification process, is added to the bias current of the other laser.

We consider each solitary laser described by the usual single-mode semiconductor laser rate equations. The bidirectional optoelectronic coupling is accounted for by adding, in the carrier rate equations, the delayed photocurrent generated by a laser into the injection current of its counterpart. The optical phase does not play any role due to the insensitivity of the photodetectors to the phase of the electrical field. Hence, the rate equations for the evolution of the photon and carrier densities in both lasers read [8]

$$
\begin{gathered}
\frac{d S_{1}}{d t}=\left(\Gamma g_{1}-\gamma_{c_{1}}\right) S_{1}, \\
\frac{d N_{1}}{d t}=\frac{J_{1}}{e d}+\xi_{c 1} S_{2}\left(t-T_{2}\right)-\gamma_{s_{1}} N_{1}-\mathrm{g}_{1} S_{1}, \\
\frac{d S_{2}}{d t}=\left(\Gamma g_{2}-\gamma_{c_{2}}\right) S_{2}, \\
\frac{d N_{2}}{d t}=\frac{J_{2}}{e d}+\xi_{c 2} S_{1}\left(t-T_{1}\right)-\gamma_{s_{2}} N_{2}-\mathrm{g}_{2} S_{2},
\end{gathered}
$$

where $S_{1,2}$ is the intracavity photon density, $N_{1,2}$ is the carrier density, and $\mathrm{g}_{1,2}$ is the material gain. The subindices 1,2 distinguish between the two lasers. $\xi_{c 1,2}$ stand for the coupling strengths that are proportional to the responsivity of the photodetectors and the amplification factor of the respective amplifiers. Our definition for the coupling coefficient is the one typically used when modeling coupled systems. However, this definition is slightly different from the one used in previous papers $[8,14]$ which is easier to determine experimentally. $T_{1,2}$ are the coupling delay times. Other parameters appearing in the rate equations are the bias current density $J$, the cavity decay rate $\gamma_{c}$, the spontaneous carrier relaxation rate $\gamma_{s}$, the confinement factor of the laser waveguide $\Gamma$, the electron charge $e$, and the active layer thickness $d$. For simplicity, spontaneous emission noise sources have been neglected. It is also important to note that an infinite-bandwith photodetector-amplifier response is assumed.
Numerical calculations and experimental measurements show that, in a wide operation range, the material gain has a linear dependence on both the carrier and photon densities. Therefore, $\mathrm{g}(N, S)$ is expanded as

$$
\mathrm{g} \approx \mathrm{g}_{0}+\mathrm{g}_{n}\left(N-N_{0}\right)+\mathrm{g}_{p}\left(S-S_{0}\right)
$$

where $\mathrm{g}_{0}=\gamma_{c} / \Gamma$ is the material gain at the solitary threshold, $\mathrm{g}_{n}=\partial \mathrm{g} / \partial N>0$ is the differential gain parameter, $\mathrm{g}_{p}$ $=\partial \mathrm{g} / \partial S<0$ is the nonlinear gain parameter, $N_{0}$ is the carrier density at threshold, and $S_{0}$ is the free-running intracavity photon density when the lasers are decoupled. The parameters $\mathrm{g}_{n}$ and $\mathrm{g}_{p}$ are taken to be approximately constants.

After introducing Eq. (5) into Eqs. (1)-(4) and defining the dimensionless variables $\tilde{s} \equiv\left(S-S_{0}\right) / S_{0}, \tilde{n} \equiv\left(N-N_{0}\right) / N_{0}$, $\tilde{J}=\left(J / e d-\gamma_{s} N_{0}\right) / \gamma_{s} N_{0}$, and $\kappa_{c} \equiv \xi_{c} \Gamma / \gamma_{c}$, the rate equations read

$$
\begin{aligned}
& \frac{d \tilde{s}_{1}}{d t}=\frac{\gamma_{c_{1}} \gamma_{n_{1}}}{\gamma_{s_{1}} \widetilde{J}_{1}} \widetilde{n}_{1}\left(\widetilde{s}_{1}+1\right)-\gamma_{p_{1}} \widetilde{s}_{1}\left(\widetilde{s}_{1}+1\right), \\
& \frac{d \tilde{n}_{1}}{d t}=\frac{\gamma_{s_{1}} \gamma_{p_{1}}}{\gamma_{c_{1}}} \widetilde{J}_{1} \tilde{s}_{1}\left(1+\tilde{s}_{1}\right)+\gamma_{s_{1}} \kappa_{c_{1}} \widetilde{J}_{1}\left[1+\tilde{s}_{2}\left(t-T_{1}\right)\right]-\gamma_{s_{1}} \tilde{n}_{1} \\
& -\gamma_{s_{1}} \widetilde{J}_{1} \tilde{s}_{1}-\gamma_{n_{1}} \tilde{n}_{1}\left(1+\tilde{s}_{1}\right), \\
& \frac{d \widetilde{s}_{2}}{d t}=\frac{\gamma_{c_{2}} \gamma_{n_{2}}}{\gamma_{s_{2}} \widetilde{J}_{2}} \tilde{n}_{2}\left(\widetilde{s}_{2}+1\right)-\gamma_{p_{2}} \widetilde{s}_{2}\left(\widetilde{s}_{2}+1\right), \\
& \frac{d \tilde{n}_{2}}{d t}=\frac{\gamma_{s_{2}} \gamma_{p_{2}}}{\gamma_{c_{2}}} \widetilde{J}_{2} \widetilde{s}_{2}\left(1+\tilde{s}_{2}\right)+\gamma_{s_{2}} \kappa_{c_{2}} \widetilde{J}_{2}\left[1+\tilde{s}_{1}\left(t-T_{2}\right)\right]-\gamma_{s_{2}} \tilde{n}_{2} \\
& -\gamma_{s_{2}} \tilde{J}_{2} \tilde{s}_{2}-\gamma_{n_{2}} \tilde{n}_{2}\left(1+\tilde{s}_{2}\right),
\end{aligned}
$$

where the differential and nonlinear carrier relaxation rates are defined as $\gamma_{n} \equiv \mathrm{g}_{n} S_{0}$ and $\gamma_{p} \equiv-\Gamma \mathrm{g}_{p} S_{0}$, respectively. It is worth noting that since $S_{0}=\widetilde{J} \gamma_{s} N_{0} \Gamma / \gamma_{c}$, both $\gamma_{n}$ and $\gamma_{p}$ are related to the bias current. The values of the parameters are those used in Ref. [8]-i.e., $\gamma_{c}=2.4 \times 10^{11} \mathrm{~s}^{-1}, \gamma_{s}=1.458$ $\times 10^{9} \mathrm{~s}^{-1}, \gamma_{n}=3 \widetilde{J} \times 10^{9} \mathrm{~s}^{-1}$, and $\gamma_{p}=3.6 \widetilde{J} \times 10^{9} \mathrm{~s}^{-1}$. The relaxation oscillation frequency is calculated as $f_{r}=(1 / 2 \pi)$ $\times\left(\gamma_{c} \gamma_{n}+\gamma_{p} \gamma_{s}\right)^{1 / 2}$, and the dimensionless coupling strength reads

$$
\kappa_{c}=a \frac{\eta c \alpha_{m} \Gamma}{2 n_{g} \gamma_{c}} \eta_{e x t},
$$

$a$ being the amplifier multiplication factor, $\eta$ the quantum efficiency of the photodetector, $\eta_{\text {ext }}$ a parameter that takes into account additional external losses, $c$ the speed of light in vacuum, $\alpha_{m}$ the laser facet losses, and $n_{g}$ the group refractive index. For a typical case $\left(\eta=0.5, \eta_{\text {ext }}=1, \alpha_{m}=48 \mathrm{~cm}^{-1}, n_{g}\right.$ $=3.5, \gamma_{c}=0.24 \mathrm{ps}^{-1}$, and $\left.\Gamma=0.3\right), \kappa_{c}$ is of the order of $\sim 0.1$, when $a$ is fixed to 1 . Then, the magnitude of $\kappa_{c}$ can be easily modified just by changing the amplification factor. In addition, the sign of $\kappa_{c}$ can be reversed by subtracting the generated photocurrent from the bias instead of adding it. In this 
paper, we consider both positive and negative values for the coupling strength.

\section{FIXED POINTS AND STABILITY ANALYSIS}

The fixed points of the system of equations (6)-(9) are obtained by imposing the condition $\widetilde{s}_{1}(t)=\widetilde{s}_{1 \mathrm{st}}, \widetilde{s}_{2}(t)=\widetilde{s}_{2 \mathrm{st}}$, $\widetilde{n}_{1}(t)=\widetilde{n}_{1 \mathrm{st}}$, and $\widetilde{n}_{2}(t)=\widetilde{n}_{2 \mathrm{st}}$. In order to simplify the algebraic manipulations and reduce the number of free parameters, we establish that, hereafter, all the computations are under the assumptions that the same bias current is applied to both lasers (i.e., $\left.\widetilde{J}_{1}=\widetilde{J}_{2} \equiv \widetilde{J}\right)$, a symmetric coupling $\left(\kappa_{c 1}=\kappa_{c 2}\right.$ $\equiv \kappa_{c}$ ) is considered, and identical internal parameters are taken for both lasers. Nevertheless, it is worth mentioning that even though that reduction is performed, there are still four free control parameters $\widetilde{J}, \kappa_{c}, T_{1}$, and $T_{2}$. The search of equilibria in the system reveals the existence of four fixed points. The first solution (FP1) $\widetilde{s}_{1 \mathrm{st}}=-1, \widetilde{n}_{1 \mathrm{st}}=\widetilde{J}, \widetilde{s}_{2 \mathrm{st}}=-1$, and $\tilde{n}_{2 \mathrm{st}}=\widetilde{J}$ defines the off state of both lasers. It loses its stability as soon as the bias current crosses the threshold value. The other three fixed points are

$$
\begin{gathered}
\widetilde{s}_{1 \mathrm{st}(2 \mathrm{st})}=0(-1), \\
\tilde{n}_{1 \mathrm{st}(2 \mathrm{st})}=0\left(\left(1+\kappa_{c}\right) \widetilde{J}\right), \\
\widetilde{s}_{2 \mathrm{st}(1 \mathrm{st})}=-1(0), \\
\widetilde{n}_{2 \mathrm{st}(1 \mathrm{st})}=\left(1+\kappa_{c}\right) \widetilde{J}(0), \\
\widetilde{s}_{1 \mathrm{st}}=\frac{\kappa_{c}}{\left(1-\kappa_{c}\right)+\frac{\gamma_{p} \gamma_{s}}{\gamma_{c} \gamma_{n}},} \\
\widetilde{n}_{1 \mathrm{st}}=\widetilde{J}_{\frac{\gamma_{p}}{\gamma_{s}} \gamma_{n}} \widetilde{s}_{1 \mathrm{st}}, \\
\tilde{s}_{2 \mathrm{st}}=\widetilde{s}_{1 \mathrm{st}}, \\
\tilde{n}_{2 \mathrm{st}}=\widetilde{n}_{1 \mathrm{st}} .
\end{gathered}
$$

The two fixed points (FP2 and FP3) given by Eqs. (11) correspond to the case in which one laser is emitting while the other is switched off, thus constituting the only asymmetric steady-state solutions. Unlike the case of mutually optically coupled lasers, no other nontrivial asymmetric fixed points are found $[19,20,22,23]$. Finally, Eq. (12) defines the on state of both lasers (FP4). Note that when both lasers are lasing simultaneously there is no threshold reduction since the coupling interaction is of incoherent nature.

We now proceed to study the stability of fixed points and limit cycles of the system of equations (6)-(9). This study has been partly carried out analytically and partly using the Matlab package DDE-BIFTOOL [24] which allows for the computing, continuation, and stability analysis of steady states and periodic solutions of delay differential equations (DDE's) with both constant and state-dependent delays.

The study of the linear stability of these fixed points is achieved, as is usual for delayed differential equations, by investigating the roots distribution of the following transcendental equation:

$$
\begin{gathered}
\operatorname{det}\left(-\lambda \mathbf{I}+\left.\frac{\partial \mathbf{f}}{\partial \mathbf{x}}\right|_{\mathbf{x}_{\mathrm{st}}}+\left.\frac{\partial \mathbf{f}}{\partial \mathbf{x}_{T_{1}}}\right|_{\mathbf{x}_{\mathrm{st}}} \exp \left(-\lambda T_{1}\right)\right. \\
\left.+\left.\frac{\partial \mathbf{f}}{\partial \mathbf{x}_{T_{2}}}\right|_{\mathbf{x}_{\mathrm{st}}} \exp \left(-\lambda T_{2}\right)\right)=0
\end{gathered}
$$

where $\mathbf{x} \equiv\left(\widetilde{s}_{1}, \widetilde{n}_{1}, \widetilde{s}_{2}, \widetilde{n}_{2}\right)^{\dagger}$, $\mathbf{f}$ is the flow defined on the righthand sides of Eqs. (6)-(9), and the notation $\mathbf{x}_{T}$ stands for $\mathbf{x}(t-T)$. Consequently, in the following we will focus on the study of the eigenvalues of Eq. (13) and the Floquet multipliers of the existing limit cycles. From these computations, the route to chaos will be traced out in our system.

For the configuration we are analyzing, the coupling between the two lasers introduces a delay in their interaction which allows for a variety of dynamical behaviors. The identification of these dynamical states and their domains in a given parameter space is one of the purposes of bifurcation theory. Since any local bifurcation of fixed points is associated with a change of sign in the real part of the root $\lambda$ of the characteristic equation [Eq. (13)], it is useful to separately study the cases in which this change occurs either when the root is real or when it is a complex conjugate pair.

\section{A. Real eigenvalues}

In the case of a real $\lambda$, it is simple to see that $\lambda=0$ is a solution of Eq. (13) for the steady states FP2, FP3, and FP4 whenever the coupling strength reaches the critical value

$$
\kappa_{c}^{*}=-1-\frac{\gamma_{p} \gamma_{s}}{\gamma_{c} \gamma_{n}} .
$$

The study of the complete set of eigenvalues of FP2 and FP3, together with the assumption that no other instability perturbs the state determined by FP4 (we will see later how Hopf bifurcations are forbidden for delay times short enough), allows us to identify that at the critical coupling given by Eq. (14) the three fixed points experience a simultaneous change of stability. Hence, the aforementioned asymmetric fixed points FP2 and FP3 become stable for $\kappa_{c}<\kappa_{c}^{*}$. However, we must realize that typical changes in the stability of fixed points involve collisions between steady states. The collisions between the fixed points FP2, FP3, and FP4 are strictly forbidden for the symmetric situation we are considering. It has to be noticed that only at the critical coupling strength do the stationary conditions of our system of equations (6)-(9) allow for an extra solution consisting of a continuum of fixed points (CFP), lying on the line $\widetilde{s}_{1}+\widetilde{s}_{2}$ $=-1$, that connects the other three fixed points involved in the stability flip. In Fig. 2, we represent the paths and stability of FP2, FP3, and FP4 as a function of the coupling strength. Figure 2 is generated by assuming a coupling delay time short enough (smaller than $\sim 20 \mathrm{ps}$ ) so that we can 


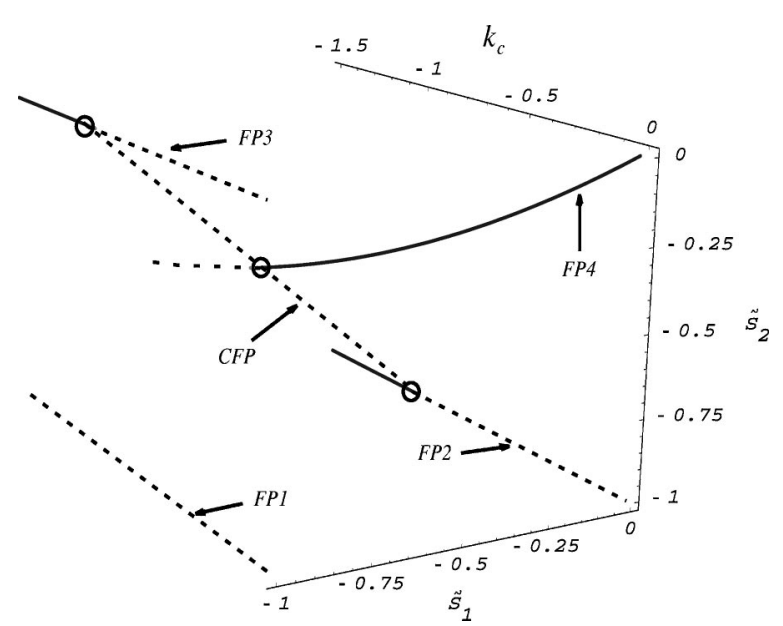

FIG. 2. Paths of the fixed points in the $\widetilde{s_{1}}-\widetilde{s}_{2}$ phase space projection as a function of the coupling strength $\kappa_{c}$. Solid and dashed lines indicate of the stable and unstable character of the fixed points, respectively. Different fixed points continuations are labeled as FP1-FP4. The continuum of fixed points (CFP) only exists for the critical coupling strength at which the stability flip ocurs.

avoid the presence of Hopf bifurcations. As we will show in the next subsection (see Fig. 5 below) the latter are forbidden for small delay times. It is observed how decreasing the coupling beyond the critical value, the symmetric FP4 becomes unstable while simultaneously FP2 and FP3 become stable nodes.

In summary, we observe that there exists a sudden transition that occurs when varying the coupling strength, from the operating regime in which both lasers are in the on state to one in which one of the lasers switches off while the other remains lasing. If we break the symmetric scenario by, e.g., allowing different coupling strengths between the two lasers, we observe that the mechanism leading to the stability flip between fixed points is again a transcritical bifurcation that this time only involves FP4 and FP2. As an example, in Fig.

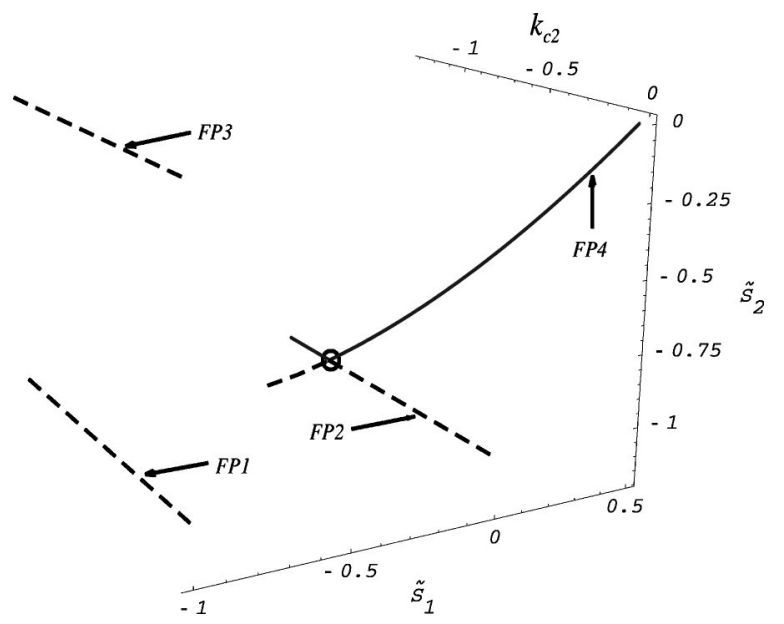

FIG. 3. Paths of the fixed points in the $\widetilde{s}_{1}-\widetilde{s}_{2}$ phase space projection as a function of the coupling strength $\kappa_{c 2} . \kappa_{c 1}$ has been fixed to 0.5 . Solid and dashed lines indicate of the stable and unstable character of the fixed points, respectively. Different fixed points continuations are labeled as FP1-FP4.

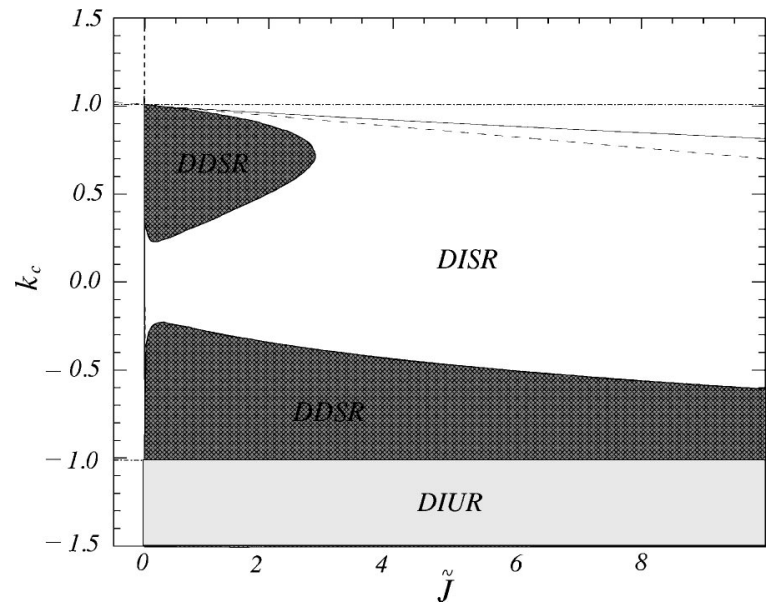

FIG. 4. Stability diagram in the coupling strength vs injection current plane $\left(\kappa_{c}-\widetilde{J}\right)$ according to the signs of $D, b$, and $c$. Solid, dashed, and dotted lines represent the zero-level contours for $D, b$, and $c$, respectively. DISR: delay-independent stable region. DIUR: delay-independent unstable region. DDSR: delay-dependent stable region.

3 , we follow the fixed points of the system when we have fixed $\kappa_{c 1}=0.5$ and $\kappa_{c 2}$ is varied. As in Fig. 2, a short enough $T$ is assumed to avoid Hopf instabilities.

\section{B. Complex eigenvalues}

The other possibility is to consider that $\lambda$ is an imaginary number. In this case the transition across the imaginary axis only occurs for the fixed point FP4 which represents the two lasers operating in the on state. Under the conditions we are imposing-i.e., at the symmetric fixed point $\widetilde{s}_{1 \mathrm{st}}=\widetilde{s}_{2 \mathrm{st}}=\widetilde{s}$ and $\widetilde{n}_{1 \mathrm{st}}=\widetilde{n}_{2 \mathrm{st}}=\widetilde{n}$ - the associated characteristic equation reads

$$
\begin{aligned}
\exp (-2 \lambda T)\left[\kappa_{c} \gamma_{c} \gamma_{n}(1+\widetilde{s})\right]^{2} & \\
= & {\left[\gamma_{p}\left(\gamma_{s}+\lambda\right)(1+2 \widetilde{s})+\lambda\left(\gamma_{n}+\gamma_{s}+\lambda+\gamma_{n} \widetilde{s}\right)\right.} \\
& \left.+\frac{\gamma_{c} \gamma_{n}}{\gamma_{s} \widetilde{J}}\left[-\lambda \widetilde{n}+\gamma_{s}(\tilde{J}-\tilde{n}+\widetilde{J} \widetilde{s})\right]\right]^{2},
\end{aligned}
$$

after defining $T \equiv\left(T_{1}+T_{2}\right) / 2$. We notice that the delay times $\left(T_{1}\right.$ and $\left.T_{2}\right)$ appear in the above equation only through their sum; thus, the actual value of each of them is not important provided their sum remains constant. The Hopf bifurcation points are obtained by substituting $\lambda=i \omega$, with $\omega \neq 0$, in Eq. (15) and separating into real and imaginary parts on both sides of the equation. In this way the delay time can be eliminated, reducing the characteristic equation to the biquadratic form

$$
\omega^{4}+b \omega^{2}+c=0
$$

where the coefficients $b$ and $c$ are given by 


$$
\begin{gathered}
b=\left(\frac{\gamma_{c} \gamma_{n} \widetilde{n}}{\gamma_{s} \widetilde{J}}\right)^{2}+\left[\gamma_{s}^{2}+\gamma_{n}^{2}(1+\widetilde{s})^{2}+\left(\gamma_{p}+2 \gamma_{p} \widetilde{s}\right)^{2}+2 \gamma_{n}(1+\widetilde{s})\left(\gamma_{p}+\gamma_{s}+2 \gamma_{p} \widetilde{s}\right)\right], \\
-\frac{2 \gamma_{c} \gamma_{n}}{\gamma_{s} \widetilde{J}}\left[\gamma_{s} \widetilde{J}(1+\widetilde{s})+\widetilde{n}\left(\gamma_{n}+\gamma_{p}+\gamma_{n} \widetilde{s}+2 \gamma_{p} \widetilde{s}\right)\right] \\
c=\frac{\left[\gamma_{p} \gamma_{s} \widetilde{J}(1+2 \widetilde{s})+\gamma_{c} \gamma_{n}(\widetilde{J}-\widetilde{n}+\widetilde{J} \widetilde{s})\right]^{2}-\left[\widetilde{J} \kappa_{c} \gamma_{c} \gamma_{n}(1+\widetilde{s})\right]^{2}}{\widetilde{J}^{2}} .
\end{gathered}
$$

The solutions of Eq. (16) are

$$
\omega= \pm \frac{1}{\sqrt{2}} \sqrt{-b \pm \sqrt{b^{2}-4 c}},
$$

which depend on the internal parameters and the operating conditions. Once the values of $\omega$ have been obtained, the critical delay time can be calculated from

$$
T^{*}(\omega)=\frac{1}{\omega} \arctan \left[-\left(\frac{\omega\left\{\gamma_{s} \widetilde{J}\left(\gamma_{p}+\gamma_{s}+2 \gamma_{p} \widetilde{s}\right)+\gamma_{n}\left[-\gamma_{c} \widetilde{n}+\gamma_{s} \widetilde{J}(1+\widetilde{s})\right]\right\}}{\gamma_{s}\left\{\gamma_{c} \gamma_{n}(\widetilde{J}-\widetilde{n}+\widetilde{J} \widetilde{s})+\widetilde{J}\left[-\omega^{2}+\gamma_{p}\left(\gamma_{s}+2 \gamma_{s} \widetilde{s}\right)\right]\right\}}\right)\right] .
$$

A simple inspection of Eq. (15) reveals that its pure imaginary solutions are periodic in $T$ due to the term $\exp (-2 i \omega T)$. It is now evident that if $T^{*}$ is a critical delay time (i.e., a delay for which an eigenvalue is crossing the imaginary axis), then any delay of the form $T=T^{*}+m \pi / \omega$ $\forall m \in \mathbb{Z}$ will also be a critical delay time. From Eq. (17) it turns out that a necessary and sufficient condition for the existence of a critical delay time can be obtained whenever the quantity $-b \pm\left(b^{2}-4 c\right)^{1 / 2}$ provides a real and positive number. Since the coefficients $b$ and $c$ only depend on the current

injection $\widetilde{J}$ and coupling strength $\kappa_{c}$, for a given set of internal parameters, we can obtain the regions in the coupling strength versus current plane where at least one real solution for $\omega$ exists. Consequently a critical delay time associated with this value of $\omega$ will always exist. Since the expression for $T(\omega)$ is invariant under the transformation $\omega \mapsto-\omega$, we only distinguish two branches of solutions depending on the sign that is chosen inside the square root: $\omega_{1}=\left\{\left[-b+\left(b^{2}\right.\right.\right.$ $\left.\left.-4 c)^{1 / 2}\right] / 2\right\}^{1 / 2}$ and $\omega_{2}=\left\{\left[-b-\left(b^{2}-4 c\right)^{1 / 2}\right] / 2\right\}^{1 / 2}$. From inspection of Eq. (17), it is observed that the region in the coupling strength-current injection parameter space for which an instability may arise (i.e., a real solution for $\omega$ exists) is delimited by the conditions $D \equiv b^{2}$ $-4 c>0 \cap[b<0 \cup(b>0 \cap c<0)]$. In that area, we can be assured that there is a value for the delay time, given by expression Eq. (18), beyond which at least one pair of complex conjugate eigenvalues have a positive real part. Note that the case $b=0$ has not been considered since it violates the condition $D \geqslant 0$ unless $c=0$ in which case both $\omega_{1}$ and $\omega_{2}$ are equal to zero.

\section{Stability diagrams}

In this section, we provide a complete overview of the dependence of the stability with respect to variations of the injection current, coupling strength, and time delay. We start our investigations by looking at the injection current versus coupling strength parameter space. In Fig. 4, we show the stability diagram of FP4 according to the conditions $\lambda$ being zero or a purely imaginary number. We can identify three different regions: the delay-independent stable region (DISR), the delay-independent unstable region (DIUR), and two delay-dependent stable regions (DDSR). In the DISR zone, no matter the delay times we chose, the system will remain operating in its symmetric steady state given by FP4. In the DIUR zone, independently of the delay times the FP4 is unstable, while the FP2 and FP3 become stable. Finally, in the DDSR zone the stability of the symmetric fixed point is not only determined by the injection current and the coupling

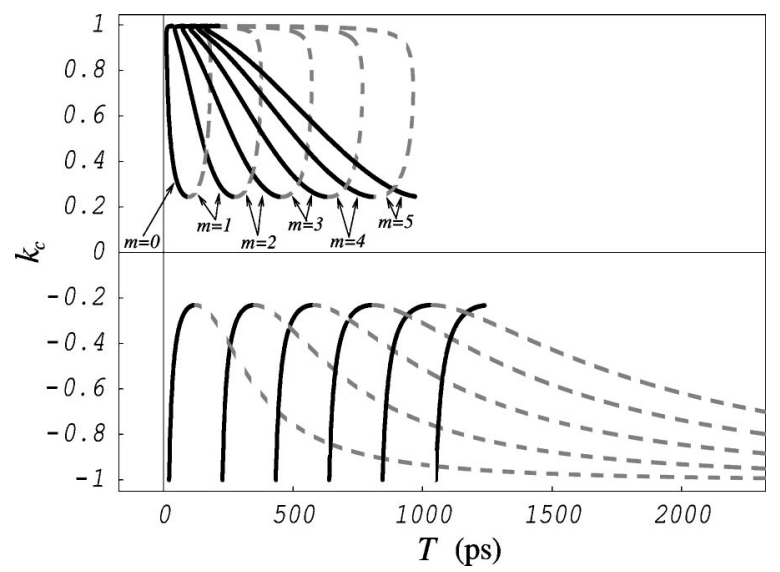

FIG. 5. Hopf curves $T^{*}\left(\omega_{1,2}\right)$ with $m=0,1,2,3,4$, and 5 in the coupling strength vs time delay plane. Solid and dashed lines distinguish between the sequences associated with $\omega_{1}$ and $\omega_{2}$, respectively. The Hopf curve associated to $T^{*}\left(\omega_{2}\right)$ with $m=0$ has not been considered here, since it appears at negative couping delay times. 
strength but also by the exact value of the coupling delay time. The size of each of these former regions may strongly depend on the nonlinear carrier relaxation rate parameter $\gamma_{p}$. For instance, we have observed that an increase of the saturation yields a reduction of the size of DDSR island corresponding to positive couplings. Hence, the nonlinear gain parameter, besides introducing an essential nonlinearity in the system, becomes an important laser parameter that tends to stabilize the dynamics by reducing the areas where instabilities may appear. Another remarkable feature of these stability diagrams is the fact that it is easier to destabilize the system through inhibitory couplings than through excitatory ones. This can be understood by noting that the damping of the relaxation oscillations decreases for negative coupling since the effective injection current is reduced. It is also worth noting the existence, for any fixed coupling, of a maximum bias beyond which any dynamical instability is strictly forbidden. Only for the DIUR zone can stability flip be induced below the critical coupling for an arbitrarily large current.

In order to better understand the effect of the time delay on the stability properties, we fix the value of the bias current to $\widetilde{J}=1 / 3$. From Fig. 4 , it can be seen that the minimum positive coupling which can lead to an instability is $\kappa_{c}$ $\sim 0.25$, while for negative couplings the minimum is $\kappa_{c}$ $\sim-0.23$. To construct the stability diagram in the coupling strength versus time delay plane we have to compute the critical delay times given by Eq. (18) for different values of $m$, taking into account that the real solutions for $\omega$ come in pairs of different signs. This allows us to distinguish among only two positive eigenfrequencies $\omega_{1}$ and $\omega_{2}$, and consequently two families of curves for $T^{*}(\omega)$ are obtained. Figure 5 shows the critical delay time curves corresponding to both eigenfrequencies, for values of $m$ ranging from 0 to 5 .

Besides the information provided by the critical delay time curves, we also need to check where the crossing of the eigenvalues is from the left to the right half-plane of the complex plane, in order to construct the stability diagram. This is achieved by evaluating the derivative of the eigenvalues when they cross the imaginary axis. The implicit derivative of Eq. (15) with respect to $T$ leads to the expression

$$
\frac{d \lambda}{d T}=\frac{-\lambda \exp (-2 \lambda T)}{h(\lambda) \frac{\partial h}{\partial \lambda}+T \exp (-2 \lambda T)},
$$

where $h(\lambda)$ denotes the function

$$
\begin{aligned}
h(\lambda)= & {\left[\kappa_{c} \gamma_{c} \gamma_{n}\left(\gamma_{c} \gamma_{n}+\gamma_{p} \gamma_{s}\right)\right]^{-1}\left\{\left(1-2 \kappa_{c}\right) \gamma_{c}^{2} \gamma_{n}^{2}+2 \gamma_{c} \gamma_{n} \gamma_{p} \gamma_{s}\right.} \\
& +\gamma_{p}^{2} \gamma_{s}^{2}+\lambda^{2}\left[\gamma_{c} \gamma_{n}\left(1-\kappa_{c}\right)+\gamma_{p} \gamma_{s}\right]+\lambda\left[\gamma _ { p } \gamma _ { s } \left(\gamma_{n}+\gamma_{p}\right.\right. \\
& \left.\left.\left.+\gamma_{s}\right)+\gamma_{c} \gamma_{n}\left(\gamma_{n}+\gamma_{p}+\gamma_{s}-\kappa_{c} \gamma_{s}\right)\right]\right\} .
\end{aligned}
$$

We have numerically evaluated $d \operatorname{Re}(\lambda) /\left.d T\right|_{\lambda=i \omega_{1,2}}$ from Eq. (19) as function of $m$ for several coupling constants. The results show that for all the values of $\kappa_{c}$ and $m$ investigated, $d \operatorname{Re}(\lambda) /\left.d T\right|_{\lambda=i \omega_{1}}>0$, while $d \operatorname{Re}(\lambda) /\left.d T\right|_{\lambda=i \omega_{2}}<0$. This indicates that the destabilization of the eigenvalues occurs at the critical delay lines associated with the eigenfrequency $\omega_{1}$ while stabilizations occur at the lines associated with $\omega_{2}$.

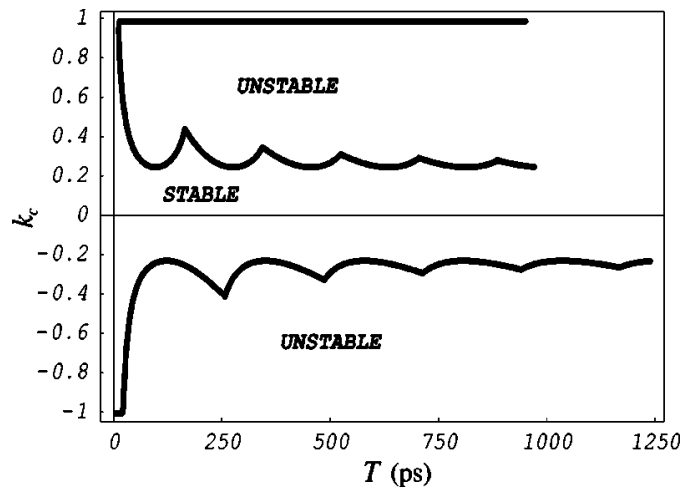

FIG. 6. Stability diagram of the FP4 in the coupling strength vs time delay plane obtained from margins of the critical lines in Fig. 5 and the critical line $\kappa_{c}^{*}=-1-\gamma_{p} \gamma_{s} / \gamma_{c} \gamma_{n}$.

This result, together with the fact that $\pi / \omega_{1}<\pi / \omega_{2}$, demonstrates that the rate at which the eigenvalues become unstable at $\omega_{1}$ is larger than the rate they become stable at $\omega_{2}$. Consequently, an arbitrarily large number of unstable eigenvalues can be achieved for sufficiently long delay times. At this point, we can now guarantee the absence of stability islands inside the external borders of the curves plotted in Fig. 5. The resulting stability diagram for FP4 is shown in Fig. 6. We further continue our study by looking at the periodic solutions and the route to chaos the system can undergo.

\section{PERIODIC SOLUTIONS AND THE ROUTE TO CHAOS}

In the preceding section we showed that the system can be destabilized through a Hopf bifurcation at frequency $\omega_{1,2}$, leading to the appearance of oscillations at the same frequency. Now, we study the evolution of the eigenfrequencies $\omega_{1,2}$ as a function of the coupling strength, and we compare these values with the relaxation oscillation frequency (ROF) computed using the effective injection current (bias plus photocurrent). Figure 7 illustrates that the transition of the system from stable to pulsating behavior occurs at a nearly constant frequency that is close to the free-running ROF $(2.47 \mathrm{GHz})$ when negative coupling is considered. For positive values, $f_{1}$ grows above the ROF of the coupled system until it reaches a maximum around $\kappa_{c} \sim 0.98$. Concerning the frequency $f_{2}$, it is observed that when stabilization of the output of the lasers occurs, it is through an inverse Hopf bifurcation at a frequency almost identical to the freerunning $\mathrm{ROF}$ for positive couplings values and at a lower frequency for negative ones.

Next, we analyze the structure and stability of the limit cycles embedded in the phase space in the route to chaos followed by the system. In particular, we are interested in understanding how a typical bifurcation diagram, like that shown in Fig. 8 for the normalized photon density $\widetilde{s}_{1}$, develops in terms of the periodic solutions and their interactions. We will see how the main features of Fig. 8-namely, (a) the sudden transitions from chaos $(C)$ to periodic behavior $(P)$, (b) the increasing size of the quasiperiodic and chaotic regions ( $Q$ and $C$, respectively), and (c) the clear repetitive 


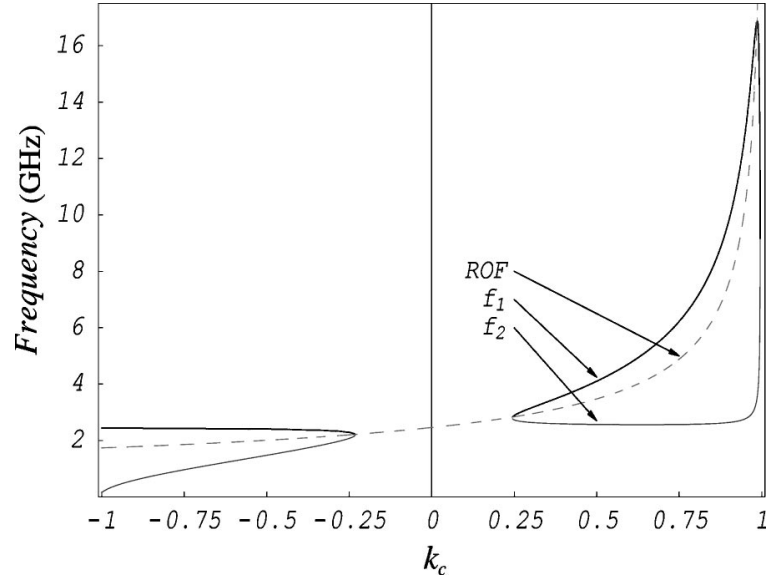

FIG. 7. Eigenfrequencies $f_{1,2}$ (solid lines) and ROF (dashed line) as a function of the coupling strength.

structure of this diagram - are nothing else but the signature of the properties of the limit cycles on the dynamics of the system. In Fig. 8 one can also observe that the route to chaos traced by our mutually interacting lasers resembles the one followed by a single laser subject to optoelectronic feedback [8].

The top panel of Fig. 9 shows the stability diagram for the limit cycles as a function of the coupling delay time. We typically observe an unstable Hopf point (A) [25-27] giving rise to a limit cycle, which undergoes a stabilizing fold bifurcation $(B)$. This fold bifurcation is followed by a Neimark-Sacker $(C)$ bifurcation. At this point the limit cycle in which the system is operating developes into a torus and a quasiperiodic dynamical state emerges. When further decreasing the time delay, a torus breakdown is generally observed, leading to fully developed chaos. Besides the numerical evidence of this torus breakdown, a parametric representation of the system defined by Eqs. (6)-(9) can be used to continue the quasiperiodic solutions when a parameter is varied, as has been done in Refs. [19,25,28]. This

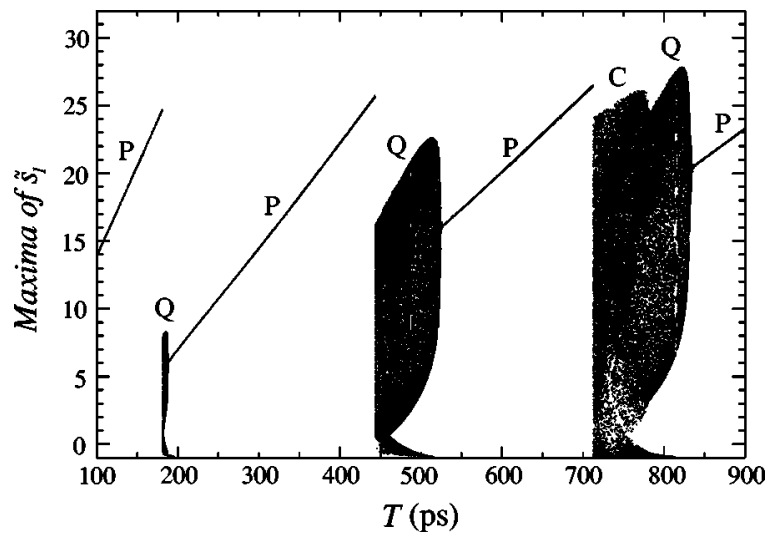

FIG. 8. Bifurcation diagram for $\widetilde{s}_{1}$ as a function of the coupling delay time. The coupling strength has been fixed to $\kappa_{c}=0.5$. Only maxima of time series were recorded to plot the bifurcation diagram. The labels $P, Q$, and $C$ on the top of the diagram identificate the dynamical states as periodic, quasiperiodic, and chaotic, respectively.
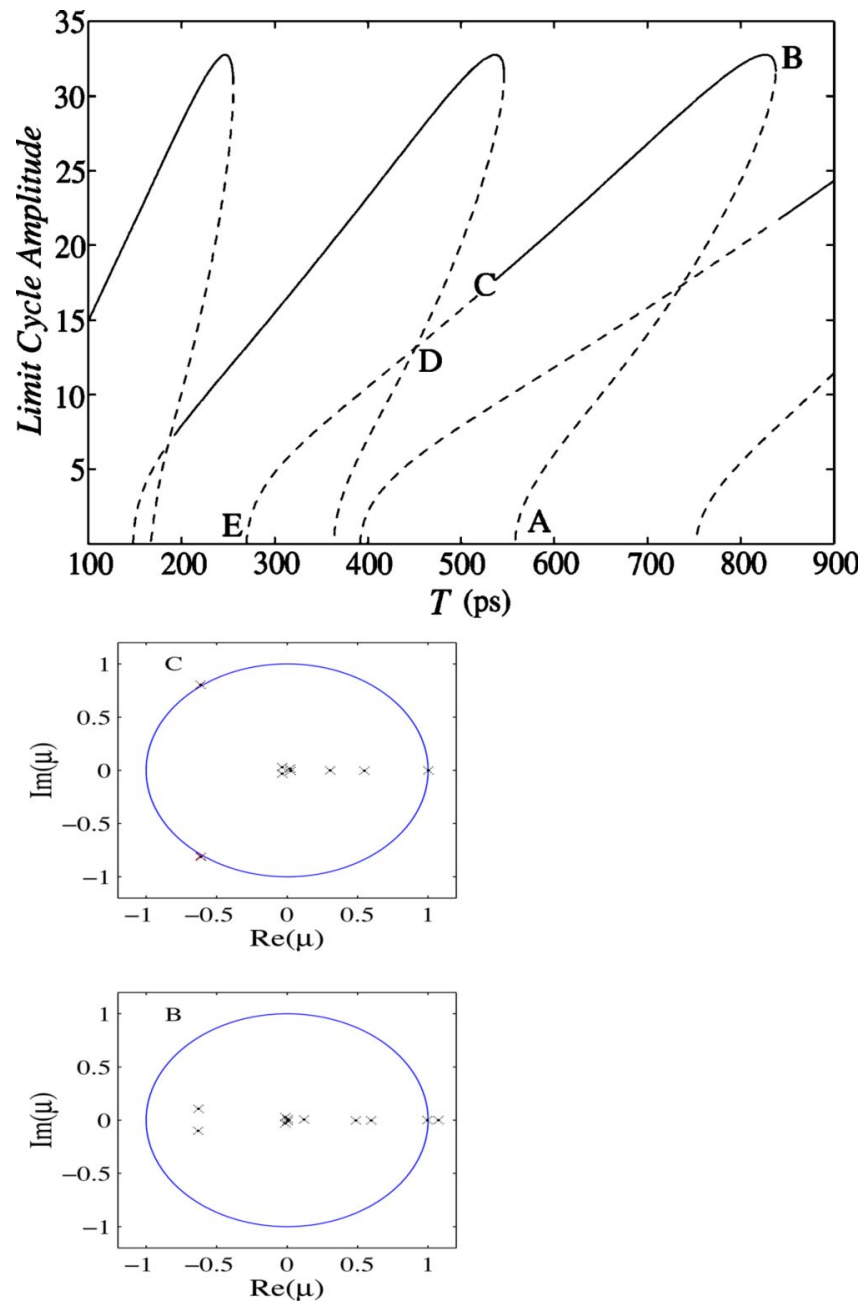

FIG. 9. Top panel: stability diagram for limit cycles as a function of the coupling delay time. The coupling strength has been fixed to $\kappa_{c}=0.5$. Solid and dashed lines indicate stability and instability, respectively. Bottom panel: Floquet multipliers at the points labeled as "B" and "C" in the top panel.

route to chaos is rigorously checked through the computation of the Floquet multipliers (i.e., the eigenvalues of the monodromic equation for limit cycles which give information about their stability) at the appropriate points as is illustrated in the bottom panel of Fig. 9. At the point labeled as $(B)$, we observe how a real Floquet multiplier is entering into the unit circle through the $(1,0)$ coordinate, while for the point $(C)$ two complex conjugate Floquet multipliers are leaving the unit circle, giving rise to a toroidal structure. For even shorter delays, the sudden disappearance of the chaotic behavior observed in Fig. 8 might be induced by a boundary crisis $(D)$ that occurs when another unstable limit cycle collides with the chaotic attractor that was born around the torus. Finally, the amplitude of the periodic solution goes to zero $(E)$. Since the limit cycle is born $(A)$ and annihilated $(E)$ on FP4, the periodic orbit connects different points on the continuation path of FP4. Similar periodic orbit bridges but between different steady states have been reported in the literature of semiconductor lasers subject to optical feedback $[26,27,29]$. 


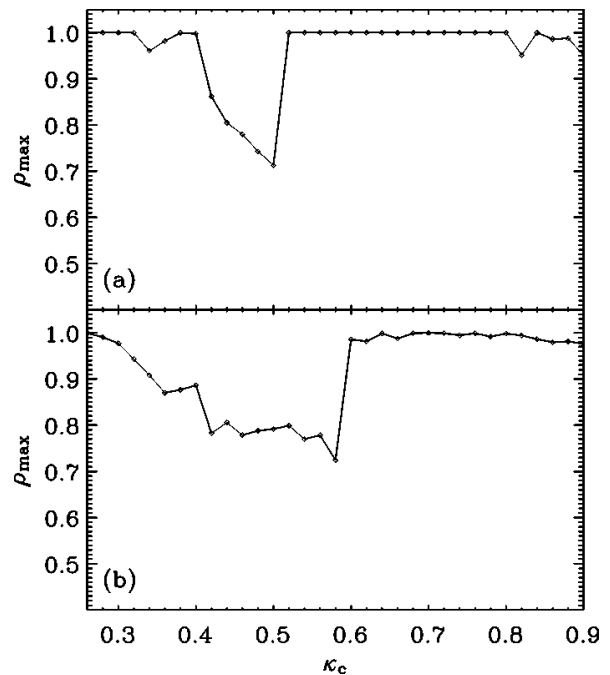

FIG. 10. Maximum of the cross-correlation function $\left(\rho_{\max }\right)$ as a function of the coupling strength for (a) $T=1 \mathrm{~ns}$ and (b) $T=15 \mathrm{~ns}$.

The above explanation was given for the limit cycle that covers the delay times in the range $\sim 440-720 \mathrm{ps}$. Now, taken into account that any other limit cycle in Fig. 9 has similar properties to the one we described, we can easily understand that (a) all the sudden transitions from complex to regular behavior are probably induced by crisis events, (b) the size of the islands of quasiperiodic or chaotic behavior amounts to the distance between points $C$ and $D$ which increases for longer delay times, and (c) the qualitatively repetitive structure found in Fig. 8 comes from the fact that new periodic solutions with similar properties arise as the delay is increased.

Once the different dynamical states have been characterized and the transition from regular to complex behavior is understood, it is interesting to know whether the lasers are able to mutual entrain their dynamics or not. To study this, we computed the maximum of the cross-correlation function $\left(\rho_{\max }\right)$ between the two outputs as a function of the coupling strength, for a short (1 ns) and a long (15 ns) delay time. Only values of $\kappa_{c} \gtrsim 0.25$ are considered since the system operates in a stable regime for smaller values. In Fig. 10, it can be seen that for short $T$ a large correlation coefficient is obtained for almost any value of the coupling coefficient we have considered. However, for most of the coupling coefficients both lasers operate in a periodic regime exhibiting synchronous (zero-lagged) pulsations. However, we noticed that changing the time delay around $\sim 1 \mathrm{~ns}$, antiphase oscillations can also be observed for almost any coupling coefficient. Only for $\kappa_{c}$ around $\sim 0.5$ do the lasers operate in a quasiperiodic or chaotic regime, and in these cases the maximum of the cross-correlation coefficient drops to around $\sim 0.7$. When increasing the delay time up to $15 \mathrm{~ns}$, we observe that for almost any coupling coefficient both lasers enter into a quasiperiodic regime or even into a chaotic pulsating state for intermediate couplings. In this case, the maximum of the cross-correlation coefficient remains close to 1 except for intermediate-coupling values at which it decays to $\sim 0.8$. Now, the time lag at which this maximum is found always corresponds to $\sim \pm T$. Contrary to the previous case,
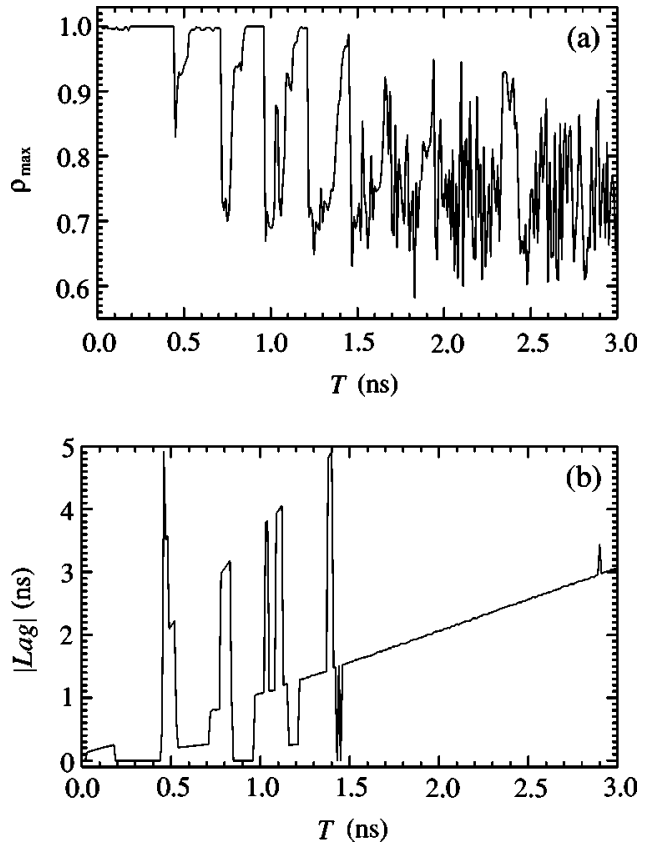

FIG. 11. (a) Maximum of the cross correlation $\left(\rho_{\max }\right)$ as a function of the coupling delay time $T$. (b) Absolute value of the lag at which the maximum of the cross-correlation function occurs. In both cases the coupling strength has been fixed to $\kappa_{c}=0.5$.

now changing the delay time (within the long-delay-time limit), the lag at which the maximum of the correlation appears continues to be located at $\sim \pm T$ and no zero-lagged solutions are found. When reversing the sign of the coupling coefficient, in order to take into account negative coupling values, we found that the cross-correlation function decays to much smaller values than for its positive coupling counterparts, revealing that mutual entrainment is more difficult to achieve in the case that inhibitory couplings are considered.

In order to further study the relative dynamics between the two lasers and its dependence on the coupling delay time, the maximum of the cross-correlation function and the time lag of this maximum are investigated. The results are presented in Fig. 11 for a fixed coupling strength of $\kappa_{c}=0.5$. Figure 11(a) shows the maximum correlation between the two laser outputs. It can be clearly seen that the correlation decreases from a value near 1 , for short $T$, to a value around 0.8 for a large $T$. We also observed that the high correlation is only obtained when the system operates in a limit cycle while it decreases when the system operates in a chaotic regime. In Fig. 11(b) we plot the absolute value of the lag at which the maximum of the correlation appears. We have to stress that in all cases the cross correlation is a symmetric function of the lag, which indicates that no leader-laggard dynamics takes place. For short $T$ some windows of in-phase and antiphase dynamics, corresponding to the operation of both lasers in several limit cycles, are observed. There are also some windows where the lag between series is larger than the coupling delay time $T$. These regimes mainly correspond to situations where both lasers operate in a quasiperiodic orbit. After these windows the lag between the two outputs tends to the coupling delay time $T$ for large values of $T$, where the system operates in a chaotic regime. The relative 


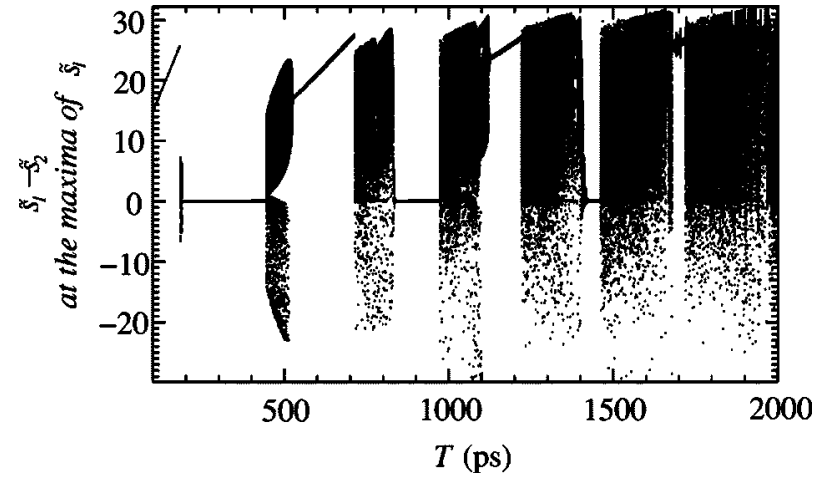

FIG. 12. Bifurcation diagram of $\widetilde{s_{1}}-\widetilde{s}_{2}$ at the maxima of $\widetilde{s}_{1}$, when the coupling delay time $T$ is varied. The coupling strength has been fixed to $\kappa_{c}=0.5$.

dynamics between the two lasers allows us to represent, in Fig. 12, the bifurcation diagram obtained by recording the value of $\widetilde{s_{1}}-\widetilde{s_{2}}$ at the time when $\widetilde{s_{1}}$ goes through a maximum. In this figure, we observe again that for small delays the regions of the in-phase and antiphase dynamics between the lasers are interrupted by quasiperiodic or chaotic windows until they disappear for large values of $T$. The in-phase or antiphase dynamics of the system is associated with alternating states of limit cycle, when the latter are ordered from small to large values of $T$. By operating the system in the first limit cycle (starting from the left) of Fig. 9 we obtain an antiphase dynamics between the two lasers, while at the second limit cycle an in-phase dynamics is observed. Again, an antiphase dynamics appears for the third limit cycle, an inphase for the fourth one, and so on.

As a final example, we illustrate in Fig. 13 the route to chaos obtained from the numerical simulations when the coupling delay time is decreased. Temporal traces, power spectra, and return maps clearly indicate a quasiperiodic route to chaos that perfectly agrees with the theoretical analysis and is also experimentally confirmed in the following section. In the first row, a perfectly periodic state is observed for a delay time of $T=840 \mathrm{ps}$, giving rise to a single peak in the power spectrum near the ROF and a single spot in the return map plot. Decreasing the delay down to $T$ $=800 \mathrm{ps}$, the quasiperiodic state is revealed by the power spectrum. There, a slow frequency corresponding to the envelop frequency, a fast frequency coinciding with the pulsating frequency, and several harmonics and beatings between the fast and slow frequencies are clearly observed. The annular-shaped return map also confirms the quasiperiodic behavior. Finally, for a delay of $T=720 \mathrm{ps}$, a chaotically pulsating sequence is obtained. In this case, a broader spectrum and return map are expected as can be checked in the figure.

\section{EXPERIMENTAL RESULTS}

In the experiments, the lasers are In-Ga-As-P/InP singlemode DFB lasers both operating at $1.299 \mu \mathrm{m}$ wavelength and temperature stabilized at $21^{\circ} \mathrm{C}$. The two lasers, which are chosen from the same wafer, are closely matched in their characteristics to be highly identical. The photodetectors are InGaAs photodetectors with a 6-GHz bandwidth, and the amplifiers are Avantek SSF86 amplifiers with $0.4-3 \mathrm{GHz}$ bandpass characteristics. The laser intensities measured by the photodetectors are recorded with a Tektronix TDS 694C digitizing sampling oscilloscope with a $3-\mathrm{GHz}$ bandwidth and a sampling rate up to $1 \times 10^{10}$ samples/s. Power spectra are measured with an HP E4407B rf spectrum analyzer that has a spectral range from $9 \mathrm{kHz}$ to $26.5 \mathrm{GHz}$. The mutual coupling strength and the coupling delay time can be adjusted by changing the attenuation on the coupled optical power and the optical path length in the coupling channel, respectively.

Figure 14 shows a sequence of three dynamical states which are regular pulsing (RP), two-frequency quasiperiodic pulsing $(\mathrm{Q} 2)$, and chaotic pulsing $(\mathrm{C})$, respectively, obtained by varying the coupling delay times around $T_{1}=T_{2} \sim 15 \mathrm{~ns}$. Although the coupling strength is experimentally difficult to measure, we estimate it to be between 0.5 and 1 . For each dynamical state, the time series, power spectrum, and return map from the system output at photodetector PD2 are plotted as in the first, second, and third columns, respectively. The output of the system from PD1 is similar to that from PD2 for each dynamical state.
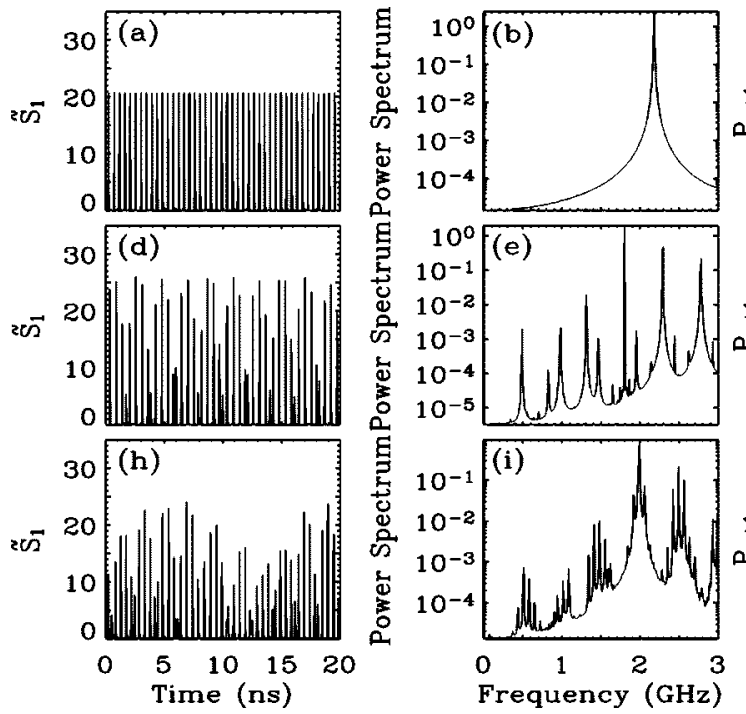

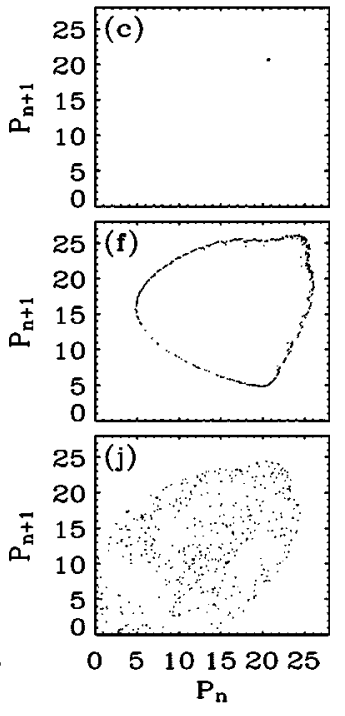

FIG. 13. Numerically computed quasiperiodic route to chaos. Left column, time series; central column, power spectra; right column, return maps. From top to bottom the delay time $T$ is $840 \mathrm{ps}, 800 \mathrm{ps}$, and $720 \mathrm{ps}$, respectively. The coupling strength is $\kappa_{c}=0.5$. 

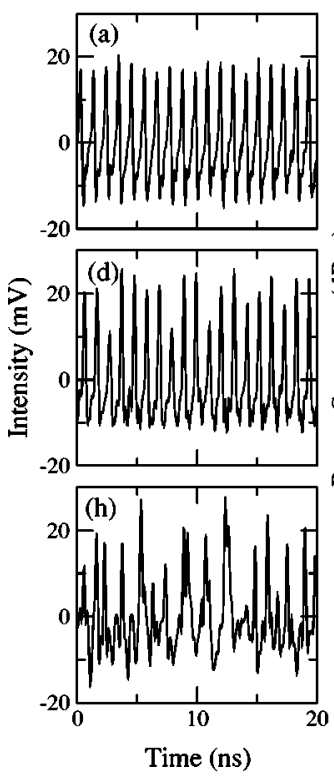
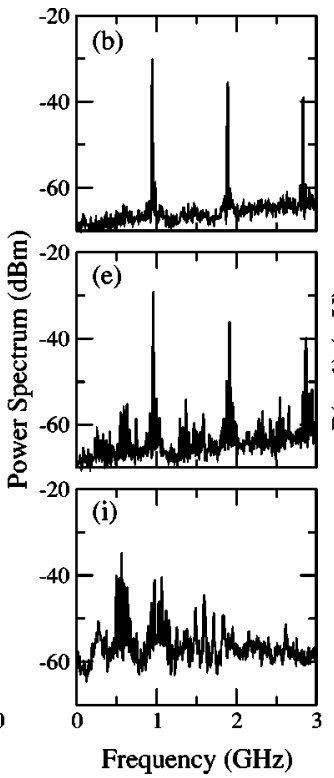

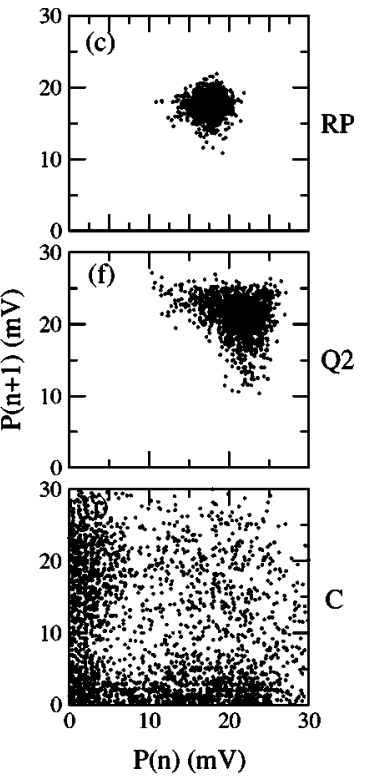

FIG. 14. Experimental quasiperiodic pulsing route to chaos when decreasing the coupling delay time (from top to bottom) for mutually coupled lasers with the configuration shown in Fig. 1. RP, regular pulsing state; Q2, twofrequency quasiperiodic pulsing state; $\mathrm{C}$, chaotic pulsing state. First column, time series; second column, power spectra; third column, return maps.
In Figs. 14(a)-14(c), the system is in a regular pulsing state. The time series in Fig. 14(a) shows a train of regular pulses with a constant pulsing intensity and interval. The power spectrum in Fig.14(a) has only one fundamental pulsing frequency $f_{1}$, which is about $1 \mathrm{GHz}$, close to the ROF. The Poincaré map section in Fig. 14(c) is obtained by recording a peak sequence $P(n)$ at the local intensity maxima of a pulse train and further plotting $P(n)$ versus $P(n+1)$, as was done for the numerical results. In the regular pulsing state, the output has a constant peak intensity, and the return map shows only one spot. The fluctuations in the time series and, consequently, the scattering in the return map are mainly caused by the noise in the system and the sampling errors from the oscilloscope. When the coupling delay time is decreased, the system enters into a two-frequency quasiperiodic pulsing state with the pulsing intensity modulated at a frequency $f_{2}$ as shown in Figs. 14(d)-14(f). The time series clearly shows the modulation of the peak intensity. In the power spectrum, besides the pulsing frequency $f_{1}$, an incommensurate $f_{2}$ indicating the modulation of peak intensity shows up. The appearance of two incommensurate frequencies $f_{1}$ and $f_{2}$ is the indication of quasiperiodicity. In the return map, the data points are still scattered due to noise and sampling errors. However, we can see that the distribution in Fig. 14(f) is more scattered than that in Fig. 14(c) because of the modulation on the pulse intensity.
In Figs. 14(h)-14(j), when the delay is further decreased, the system enters into a chaotic pulsing state. From the time series, we find that both the pulse intensity and the pulsing interval vary chaotically. At the same time, the power spectrum of the chaotic pulsing state is broadened with a much increased background, indicating the onset of chaos. The return map shows a highly scattered distribution in a large area. Therefore, the system is shown to enter a chaotic pulsing state through a quasiperiodic route.

We have also experimentally observed that for the longdelay-time limit, the maximum of the cross-correlation function appears to be located at $\sim \pm T$, as happens in the numerical analysis. Figure 15 shows experimental temporal traces and the cross correlation between these two series. Although the correlation value is significantly lower (mainly due to noise originated in the sampling process) than the one predicted numerically ( $\sim 0.8$ for this operation regime), the two largest maxima still appear at the lags $\sim \pm T$. These experimental results verify qualitatively the results obtained from the numerical simulations.

\section{CONCLUSIONS}

In conclusion, we have extensively studied, mainly analytically and numerically, the stability diagrams of a system composed by two single-mode semiconductor lasers with bi-
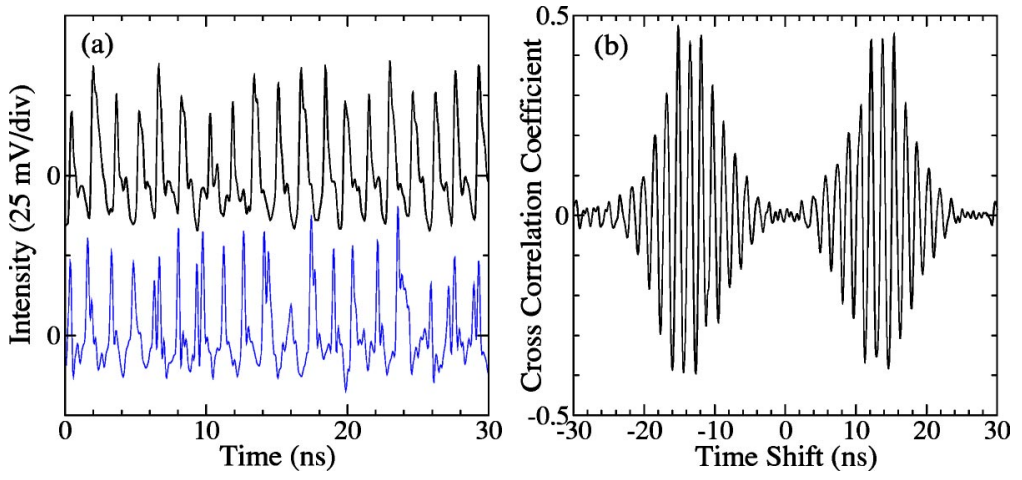

FIG. 15. Experimental temporal series and their cross-correlation function in the long-delaytime limit. The coupling delay time is $T=15 \mathrm{~ns}$. 
directional optoelectronic coupling. Our analysis provides a clear understanding of the mechanisms leading to the different dynamical instabilities and the role played by the different parameters-namely, injection current, coupling strength, and delay time. The route to chaos, when varying the coupling delay time, has been identified as a quasiperiodic scenario with crisis events. The effect of the coupling delay time on the mutual entrainment properties between the lasers has also been analyzed. Our experimental results qualitatively agree with the analytical and numerical analysis and confirm the predicted route to chaos followed by the system. It is worth remarking that the validity of the fixed-point stability analysis, performed in this paper for the optoelectronic coupling case, is maintained when the interaction between lasers is replaced by one of incoherent optical nature [30]. This comes from the fact that the characteristic equation [Eq. (15)], once linearized on the corresponding fixed points, leads to the same expression as that obtained in the optoelectronic case with negative coupling, after the substitution $\xi_{c, \text { inco }} \mapsto-\xi_{c, \text { opto }}$ is performed. Numerical simulations suggest that the same route to chaos and nonlinear phenomena is also shared by these two schemes. As future directions, we find interesting the study of the effect of having different delay times in each one of the coupling directions (i.e., $T_{1} \neq T_{2}$ ) in the mutual entrainment properties, as well as to investigate the effect of including feedback loops in each one of the laser systems.

An extensive and careful comparison between systems with feedback and mutually coupled systems would be of great interest once the dynamics of mutually coupled system is well understood.

\section{ACKNOWLEDGMENTS}

R.V., J.M., and C.R.M. acknowledge Emilio HernándezGarcía, Pere Colet, and Manuel Matías for fruitful discussions. They also acknowledge financial support from the Ministerio de Ciencia y Tecnología (Spain) and FEDER (Project Nos. BFM2000-1108, BFM2001-0341, BFM200012159, and BFM2002-04369) and from EC Project No. IST2000-29683 OCCULT. The work by J.M. is supported by the Spanish Consejo Superior de Investigaciones Científicas through Program No. I3P-PC2003 with social European funding. R.V. and C.R.M. are also grateful for the hospitality and support of the Electrical Engineering Department, University of California, Los Angeles. R.V. and C.R.M. are also funded by the Secretaría de Estado de Educación y Universidades, Ministerio de Educación, Cultura y Deporte, Spain. The work of S.T. and J.M.L. is supported by the U.S. Army Research Office under Contract No. DAAG55-98-1-0269.
[1] A. Pikovsky, M. Rosenblum, and J. Kurths, Synchronization: A Universal Concept Nonlinear Science (Cambridge University Press, Cambridge, England, 2002).

[2] J. D. Farmer, Physica D 4, 366 (1982).

[3] G. Giacomelli, M. Calzavara, and F. Arecchi, Opt. Commun. 74, 97 (1989).

[4] E. V. Grigorieva, H. Haken, and S. A. Kaschenko, Opt. Commun. 165, 279 (1999).

[5] C. Lee and S. Shin, Appl. Phys. Lett. 62, 922 (1992).

[6] F. Y. Lin and J. M. Liu, IEEE J. Quantum Electron. 39, 562 (2003).

[7] N. A. Loiko and A. M. Samson, Opt. Commun. 93, 66 (1992).

[8] S. Tang and J. M. Liu, IEEE J. Quantum Electron. 37, 329 (2001).

[9] H. D. I. Abarbanel, M. B. Kennel, L. Illing, S. Tang, H. F. Chen, and J. M. Liu, IEEE J. Quantum Electron. 37, 1301 (2001).

[10] J. P. Goedgebuer, P. Levy, L. Larger, C. Chen, and W. Rhodes, IEEE J. Quantum Electron. 38, 1178 (2002).

[11] M. Lee, L. Larger, V. Udaltsov, E. Genin, and J. Goedgebuer, Opt. Lett. 29, 325 (2004).

[12] S. Tang and J. M. Liu, Opt. Lett. 26, 596 (2001).

[13] Optical Chaos and Applications to Crytography edited by S. Donati and C. Mirasso [IEEE J. Quantum Electron. 38, 1138 (2002)].

[14] J. M. Liu, H. Chen, and S. Tang, IEEE J. Quantum Electron. 38, 1184 (2002).

[15] C. R. Mirasso, P. Colet, and P. Garcia-Fernandez, IEEE Photonics Technol. Lett. 8, 299 (1996).
[16] N. Fujiwara, Y. Takiguchi, and J. Ohtsubo, Opt. Lett. 28, 1677 (2003).

[17] T. Heil, I. Fischer, W. Elsasser, J. Mulet, and C. Mirasso, Phys. Rev. Lett. 86, 795 (2001).

[18] A. Hohl, A. Gavrielides, T. Erneux, and V. Kovanis, Phys. Rev. A 59, 3941 (1999).

[19] J. Javaloyes, P. Mandel, and D. Pieroux, Phys. Rev. E 67, 036201 (2003).

[20] J. Mulet, C. Masoller, and C. R. Mirasso, Phys. Rev. A 65, 063815 (2002).

[21] J. Mulet, C. Mirasso, T. Heil, and I. Fischer, J. Opt. B: Quantum Semiclassical Opt. 6, 97 (2004).

[22] H. Erzgraber, D. Lenstra, B. Krauskopf, and I. Fischer, Proc. SPIE Photon. Europe (to be published).

[23] J. Mulet, C. R. Mirasso, T. Heil, I. Fischer, and Elsasser, Proc. SPIE 4283, 293 (2001).

[24] K. Engelborghs, T. Luzyanina, and G. Samaey (unpublished).

[25] B. Haegeman, K. Engelborghs, D. Roose, D. Pieroux, and T. Erneux, Phys. Rev. E 66, 046216 (2002).

[26] D. Pieroux, T. Erneux, B. Haegeman, K. Engelborghs, and D. Roose, Phys. Rev. Lett. 87, 193901 (2001).

[27] M. Sciamanna, F. Rogister, O. Deparis, P. Megret, and M. Blondet, Opt. Lett. 27, 261 (2002).

[28] D. Pieroux and P. Mandel, Phys. Rev. E 67, 056213 (2003).

[29] M. Sciamanna, T. Erneux, F. Rogister, O. Deparis, P. Megret, and M. Blondet, Phys. Rev. A 65, 041801 (2002).

[30] F. Rogister, A. Locquet, D. Pieroux, M. Sciamanna, O. Deparis, P. Megret, and M. Blondel, Opt. Lett. 26, 1486 (2001). 